\title{
Histerectomia Vaginal versus Histerectomia Abdominal em Mulheres sem Prolapso Genital, em Maternidade-Escola do Recife: Ensaio Clínico Randomizado
}

\author{
Vaginal Hysterectomy versus Abdominal Hysterectomy in Patients without \\ Uterine prolapse: a Randomized Clinical Trial
}

Aurélio Antônio Ribeiro Costa, Melania Maria Ramos de Amorim, Telma Cursino

\section{RESUMO}

Objetivo: comparar os resultados intra e pós-operatórios em pacientes sem prolapso genital ou doenças anexiais, submetidas a histerectomia vaginal ou abdominal.

Métodos: realizou-se estudo do tipo ensaio clínico aberto, randomizado, em pacientes sem prolapso genital que se submeteram a histerectomia total, indicada por doenças benignas, no IMIP, em Recife, Pernambuco. Incluiram-se 35 pacientes, alocadas aleatoriamente em dois grupos, sendo 19 submetidas a histerectomia vaginal e 16 a histerectomia abdominal. Foram estudadas as seguintes variáveis: volume de perda sangüinea, necessidade de hemotransfusão, tempo operatório, dor pós-operatória (intensidade e uso de analgésicos), tempo de permanência hospitalar, complicações operatórias, tempo de retorno às atividades e grau de satisfação das pacientes. Para análise estatistica, utilizaram-se os testes $\chi^{2}$ de associação, exato de Fisher e Mann-Whitney para comparação dos grupos, considerando-se significativo erro alfa menor que 5\%.

Resultados: o volume de sangue perdido durante as histerectomias por via abdominal (mediana de $902 \mathrm{~mL}$ ) foi significativamente maior em relação à perda durante as histerectomias vaginais (mediana de $520 \mathrm{~mL}$ ), e nenhuma paciente neste último grupo requereu hemotransfusão, contra $19 \%$ no primeiro grupo. A duração da cirurgia foi semelhante, com mediana de 120 minutos nos dois grupos. A intensidade da dor, verificada pelos escores da escala analógica visual, foi significativamente menor entre as pacientes submetidas a histerectomia vaginal, que também apresentaram menor freqüência de utilização de analgésicos. Não houve diferença na freqüência de complicações intra ou pós-operatórias entre os dois grupos, encontrando-se apenas um caso de infecção em cada grupo e um caso de trombose venosa profunda no grupo das histerectomias vaginais. O tempo de retorno às atividades das pacientes submetidas à histerectomia vaginal foi significativamente menor (mediana de 35 dias) em relação ao outro grupo (mediana de 40 dias). O grau de satisfação foi semelhante nos dois grupos.

Conclusões: os beneficios da histerectomia vaginal no presente estudo incluiram menor volume de perda sanguinea intra-operatória, menor intensidade da dor pós-operatória, menor freqüência de solicitação de analgésicos e menor tempo de retorno às atividades após a cirurgia. A via vaginal pode substituir com vantagens a via abdominal em pacientes com indicação de histerectomia.

PALAVRAS-CHAVE: Histerectomia abdominal. Histerectomia vaginal. Complicações da cirúrgia. Ensaio clínico.

Instituto Materno-Infantil de Pernambuco (IMIP)

Mestrado em Saúde Materno-Infantil

Correspondência:

Melania Maria Ramos de Amorim

Rua Neuza Borborema de Sousa, 300 - Bairro Santo Antônio

58103-313 - Campina Grande - Paraíba

e-mail: melamorim@uol.com.br 
Introdução

A histerectomia é uma das cirurgias ginecológicas mais realizadas nos serviços de todo o mundo. As indicações mais freqüentes são as doenças benignas (leiomiomatose uterina, endometriose, hiperplasias), ao passo que as doenças malignas representam em torno de $10 \%$ das indicações ${ }^{1}$.

Entre janeiro de 2000 e setembro de 2002 foram realizadas no Brasil cerca de 300.000 histerectomias, das quais pouco mais de 22.000 por via vaginal ${ }^{2}$. Salvo exceção de alguns poucos serviços, a tradição brasileira sempre foi de histerectomia abdominal ${ }^{3}$, conquanto nos últimos anos venha despontando aumento das indicações da cirurgia vaginal ${ }^{4,5}$. Em outros países, o interesse pela cirurgia minimamente invasiva tem levado a aumento da freqüência de histerectomias vaginais, sobretudo com o advento da histerectomia assistida por laparoscopia ${ }^{6}$.

Diversos estudos têm demonstrado vantagens da técnica vaginal, porém apenas um ensaio clínico randomizado comparou os resultados da histerectomia vaginal simples com a histerectomia abdominal ${ }^{7}$. Estudos observacionais comparando as duas técnicas evidenciam, além da recuperação mais rápida e da menor freqüência de complicações, custos bem mais baixos para o Sistema de Saúde ${ }^{8-10}$. Por outro lado, vários ensaios clínicos controlados comparando a histerectomia vaginal assistida por laparoscopia com a técnica abdominal tradicional têm sido publicados, demonstrando vantagens inequívocas como menor perda sanguínea, menor necessidade de hemotransfusão, menor taxa de complicações, menor tempo de hospitalização e recuperação pós-operatória mais rápida ${ }^{11-14}$.

Considerando que a histerectomia vaginal simples, sem intervenção laparoscópica, apresenta teoricamente as mesmas vantagens da cirurgia vídeo-assistida, sem os inconvenientes da anestesia geral, do maior tempo cirúrgico, necessidade de instrumental especializado e custos elevados, realizamos o presente estudo para comparar os resultados intra e pós-operatórios em pacientes sem prolapso uterino, submetidas a histerectomia vaginal ou abdominal em hospital público de ensino.

\section{Pacientes e Métodos}

Realizou-se ensaio clínico randomizado, aberto, para comparação da histerectomia vaginal com a histerectomia abdominal. O estudo foi realizado no Instituto Materno-Infantil de Pernambuco (IMIP), hospital-escola com programa de residência médica em Tocoginecologia, onde se realizam anualmente cerca de 500 histerectomias. A utilização da técnica vaginal para histerectomia em pacientes sem prolapso uterino teve início em 2000, após curso teórico-prático para treinamento dos cirurgiões ginecológicos. A pesquisa em tela desenvolveu-se durante os meses de março a novembro de 2002 , tendo sido o protocolo original aprovado pelo Comitê de Ética em Pesquisa da instituição.

Incluíram-se pacientes com doença benigna e idade entre 30 e 65 anos, excluindo-se os casos com prolapso genital, doenças anexiais, atraso menstrual ou gravidez confirmada, doença trofoblástica gestacional, volume uterino maior que $300 \mathrm{~cm}^{3}$ e história de duas ou mais laparotomias anteriores. Todas as pacientes foram devidamente informadas sobre o estudo e as técnicas cirúrgicas envolvidas e aquelas que concordaram em participar assinaram o termo de consentimento livre e esclarecido.

O tamanho da amostra foi calculado para testar a diferença entre os grupos (histerectomia vaginal versus abdominal), utilizando-se teste para diferença de duas médias, considerando-se nível de significância de $5 \%$ e poder de $90 \%$. As estimativas de média (390 mL) e desvio-padrão para perda sangüínea foram obtidas a partir de estudos anteriores ${ }^{15}$. Para detectar diferença de pelo menos $300 \mathrm{~mL}$ nas médias de perda sanguínea durante a cirurgia, seriam necessárias 70 pacientes. Este número foi aumentado para 76 (38 em cada grupo), prevendo-se eventuais perdas. Entretanto, quando se realizou análise interina depois de se completar o seguimento da $38^{\mathrm{a}}$ paciente, verificou-se superioridade do tratamento em um dos grupos. Considerando-se a questão ética, foi suspensa a inclusão de novas pacientes e descontinuado o estudo. Foram excluídas três destas 38 pacientes que, depois da randomização, tiveram suas indicações cirúrgicas revertidas antes da cirurgia.

A randomização foi realizada a partir de tabela de números randômicos gerada no programa Epitable, do Epi-Info 6.04d. Somente após assinatura do termo de consentimento livre e esclarecido, o pesquisador realizou a alocação da paciente em um dos dois grupos, após abertura de envelope lacrado que continha o grupo selecionado para aquela participante, garantindo-se a ocultação da alocação.

Todos os procedimentos foram realizados em bloco cirúrgico, pelos médicos-residentes do segundo ano em Tocoginecologia, auxiliados e supervisionados por preceptores, médicos da equipe, todos com título de especialista em Ginecologia e Obstetrícia e experiência com ambas as técnicas. Para 
todos os casos, utilizou-se raquianestesia, com bupivacaína pesada a 0,5\% associada a 0,1 $\mathrm{mg}$ de morfina. As técnicas adotadas para histerectomia vaginal e abdominal foram, respectivamente, a de Heaney, modificada ${ }^{16}$ e a de Richardson ${ }^{17}$.

Todas as pacientes receberam antibioticoprofilaxia com cefazolina (1 g), imediatamente antes do início da cirurgia, independente da via (abdominal ou vaginal), conforme rotina da instituição. Sondagem vesical de demora (Foley) foi mantida durante a cirurgia e por 24 horas depois. Administraram-se 5.000 UI de heparina por via subcutânea a cada 12 horas para profilaxia do tromboembolismo, até que fosse possivel a livre deambulação.

Verificou-se a duração do ato operatório, medida a partir do momento da primeira incisão, até a última sutura. O tempo da anestesia, por sua vez, foi avaliado desde a realização do bloqueio, até o momento em que a paciente era retirada da sala. A estimativa de perda sangüínea intra-operatória foi realizada por meio da pesagem de gazes e compressas utilizadas durante o ato cirúrgico.

Para avaliação da intensidade da dor pósoperatória, utilizou-se a Escala Analógica Visual, cujos escores foram mensurados com seis, $12 \mathrm{e}$ 24 horas. Nesses mesmos intervalos, observouse a necessidade de utilização de analgésicos, conforme solicitados pelas pacientes. Pesquisaramse complicações pós-operatórias, como infecção do sítio cirúrgico, definida pelos critérios do $\mathrm{CDC}$ $(1999)^{18}$, trombose venosa profunda, hematomas e ligadura de ureter. Analisou-se ainda o tempo de permanência hospitalar depois da cirurgia (em horas), o tempo de retorno às atividades habituais e o grau de satisfação das pacientes.

Definiu-se o tempo de retorno às atividades habituais como o tempo (em dias) transcorrido entre o momento da alta e o reinício das atividades de trabalho fora ou dentro do lar, e entre este momento e o reinício da atividade sexual. O grau de satisfação foi mensurado de acordo com a avaliação subjetiva de cada paciente, sobre sentir-se ou não satisfeita com a cirurgia. Ambos os parâmetros foram avaliados no retorno pós-operatório, com oito, 15, 30 e 45 dias.

A análise estatística foi realizada no programa Epi-Info 2002, de domínio público. Para comparação das variáveis numéricas, utilizou-se o teste não paramétrico de Mann-Whitney, uma vez que a maioria destas não apresentava distribuição normal. Os testes $\chi^{2}$ de associação e exato de Fisher foram utilizados para comparação das variáveis categóricas. Em todas as etapas da análise, considerou-se significativo erro alfa menor que 5\%.

\section{Resultados}

Os grupos foram similares em relação a idade, paridade, indice de massa corporal, volume uterino, freqüência de hipertensão e laparotomias prévias. Também não houve diferença significativa nas indicações cirúrgicas, apesar de tendência a maior freqüência de miomatose uterina entre as pacientes submetidas a histerectomia vaginal (Tabela 1). Não foi necessário o uso de técnicas de morcelamento em nenhum dos casos. Também não se verificou conversão de histerectomia vaginal em abdominal.

Tabela 1 - Histerectomia vaginal versus abdominal: comparação dos grupos.

\begin{tabular}{|c|c|c|c|}
\hline Características & $\begin{array}{l}\text { Histerectomia vaginal } \\
n=19\end{array}$ & $\begin{array}{l}\text { Histerectomia abdominal } \\
\qquad n=16\end{array}$ & $p$ \\
\hline Idade (mediana, variação) & $44(35-65)$ & $45(34-65)$ & 0,65 \\
\hline IMC (mediana, variação) & $27(22-41)$ & $28(20-39)$ & 0,86 \\
\hline Tamanho uterino (mediana, variação) & $146(24-300)$ & $191(44-300)$ & 0,16 \\
\hline Hipertensão (n, \%) & $8(42)$ & $4(25)$ & 0,20 \\
\hline
\end{tabular}

IMC = índice de massa corporal

A perda sanguínea foi significativamente menor em pacientes submetidas a histerectomia vaginal em relação à abdominal (mediana de 520 $\mathrm{mL}$ versus $920 \mathrm{~mL}$, respectivamente). Por outro lado, realizou-se hemotransfusão em 19\% das pacientes que se submeteram a histerectomia abdominal, o que não aconteceu em nenhum caso da cirurgia vaginal. A duração do ato cirúrgico foi semelhante em ambos os grupos, apesar de uma freqüência maior de tempo cirúrgico superior a duas horas no grupo de histerectomia abdominal. A duração do tempo de anestesia também foi semelhante nos dois grupos (Tabela 2). 
Tabela 2 - Histerectomia vaginal versus abdominal: avaliação dos parâmetros intra e pós-operatórios.

\begin{tabular}{|c|c|c|c|}
\hline Parâmetros avaliados & $\begin{array}{l}\text { Histerectomia vaginal } \\
\qquad(\mathrm{n}=19)\end{array}$ & $\begin{array}{l}\text { Histerectomia abdominal } \\
\qquad(\mathrm{n}=16)\end{array}$ & $p$ \\
\hline \multicolumn{4}{|l|}{ Perda sanguínea } \\
\hline$\leq 600 \mathrm{~mL}$ & $11(57,9 \%)$ & $1(6,2 \%)$ & \\
\hline Mediana (mL) & 520 & 902 & 0,008 \\
\hline \multicolumn{4}{|l|}{ Duração da cirurgia } \\
\hline$\leq 120$ minutos & $15(78,9 \%)$ & $9(56,2 \%)$ & \\
\hline Mediana (minutos) & 120 & 120 & 0,2 \\
\hline \multicolumn{4}{|l|}{ Duração da anestesia } \\
\hline$>130$ minutos & $8(42,1 \%)$ & $11(68,8 \%)$ & 0,11 \\
\hline$\leq 130$ minutos & $11(57,9 \%)$ & $5(31,2 \%)$ & \\
\hline Mediana (horas) & 44 & 48 & 0,02 \\
\hline \multicolumn{4}{|c|}{ Tempo de retorno às atividades habituais } \\
\hline$>40$ dias & $1(5,3 \%)$ & $8(50,0 \%)$ & 0,003 \\
\hline$\leq 40$ dias & $18(94,7 \%)$ & $8(50,0 \%)$ & \\
\hline Mediana (dias) & 35 & 40 & 0,0002 \\
\hline \% de satisfação & $18(94,7)$ & $14(87,5)$ & 0,4 \\
\hline
\end{tabular}

A freqüência de complicações intra e pósoperatórias foi reduzida e semelhante nos dois grupos. Apenas uma paciente de cada grupo apresentou infecção pós-operatória, sendo um caso de infecção incisional superficial no grupo da histerectomia abdominal e um caso de infecção de cúpula vaginal no grupo da histerectomia vaginal. Ocorreu apenas um caso de trombose venosa profunda (paciente submetida a histerectomia vaginal). Não houve formação de hematomas ou ligadura de ureter.

Verificou-se menor duração da permanência hospitalar pós-operatória no grupo da histerectomia vaginal (mediana de 44 horas) em relação à abdominal (mediana de 48 horas). O tempo de retorno às atividades corriqueiras também foi significativamente menor para as pacientes submetidas à histerectomia vaginal (mediana de 35 dias) em relação à histerectomia abdominal (mediana de 40 dias). Em cerca de $95 \%$ das pacientes submetidas à histerectomia vaginal, o retorno às atividades ocorreu em menos de 40 dias, contra $50 \%$ das pacientes submetidas à histerectomia abdominal. Obser- vou-se grau de satisfação com o procedimento cirúrgico semelhante nos dois grupos, sendo que aproximadamente $95 \%$ das pacientes submetidas a histerectomia vaginal e $87,5 \%$ daquelas submetidas a histerectomia abdominal manifestaram-se satisfeitas com a cirurgia (Tabela 2).

Analisando-se a intensidade da dor pela escala analógica visual, verificaram-se escores significativamente menores no grupo da histerectomia vaginal tanto com seis como com 12 e 24 horas de pós-operatório (mediana de zero em todas as avaliações, contra cinco, seis e 12 no grupo da histerectomia abdominal) (Tabela 3). A necessidade de analgésicos foi significativamente menor com 12 horas entre as pacientes submetidas a histerectomia vaginal $(26,0 \%) \mathrm{em}$ relação à histerectomia abdominal $(68,8 \%)$, verificando-se ainda tendência a menor freqüência de uso de analgésicos com seis e 12 horas no primeiro grupo (respectivamente 26,3 e $26,1 \%$ ) em relação ao segundo (respectivamente 56,2 e $50 \%$ ). 
Tabela 3 - Histerectomia vaginal versus abdominal: intensidade da dor pós-operatória.

\begin{tabular}{|c|c|c|c|}
\hline $\begin{array}{l}\text { Escores da escala } \\
\text { analógica visual }\end{array}$ & $\begin{array}{l}\text { Histerectomia vaginal } \\
\qquad(\mathrm{n}=19)\end{array}$ & $\begin{array}{l}\text { Histerectomia abdominal } \\
\qquad(\mathrm{n}=16)\end{array}$ & $p$ \\
\hline \multicolumn{4}{|l|}{6 horas } \\
\hline Escores 0-5 & $16(84,2 \%)$ & $8(50,0 \%)$ & \\
\hline Mediana & 0 & 5 & 0,05 \\
\hline \multicolumn{4}{|l|}{12 horas } \\
\hline Mediana & 0 & 6 & 0,01 \\
\hline \multicolumn{4}{|l|}{24 horas } \\
\hline Escores 6-10 & $1(5,3 \%)$ & $6(37,5 \%)$ & 0,04 \\
\hline Escores 0-5 & $18(94,7 \%)$ & $10(62,5 \%)$ & \\
\hline Mediana & 0 & 2 & 0,02 \\
\hline
\end{tabular}

\section{Discussão}

Os resultados deste estudo sugerem que a histerectomia total, realizada pela via vaginal para pacientes sem prolapso genital com indicação cirúrgica por doenças benignas, constitui abordagem cirúrgica com melhores resultados e menor nível de complicações intra e pós-operatórias. Mesmo em se tratando de pequeno ensaio clínico, incluindo apenas 35 pacientes, evidenciou-se menor perda sanguínea intra-operatória, menor intensidade da dor pós-operatória, menor permanência hospitalar e tempo mais rápido de retorno às atividades habituais.

Estes achados não são surpreendentes, uma vez que vários autores têm demonstrado vantagens da via vaginal sobre a abdominal para a realização de histerectomia ${ }^{4,5,19-21}$. Além da recuperação mais rápida e da menor freqüência de hemorragia e outras complicações, a via vaginal implica custos bem mais baixos para o Sistema de Saúde ${ }^{9,10}$.

Por outro lado, expressivo número de estudos não controlados ressalta a exeqüibilidade e segurança das histerectomias vaginais sem intervenção laparoscópica: em série prospectiva de 806 histerectomias por doenças benignas, na ausência de prolapso uterino, a histerectomia vaginal isolada foi possivel em $80,6 \%$ dos casos, sendo necessária a laparoscopia em apenas $9,4 \%$ e laparotomia em $10 \%$ das intervenções ${ }^{22}$.

Em nosso meio, Macedo et al. ${ }^{4}$, no Rio Grande do Norte (Natal), publicaram sua experiência com 370 histerectomias vaginais em pacientes sem prolapso uterino, descrevendo taxa de complicações menor que $4 \%$. A média de tempo cirúrgico foi de 60 minutos. Figueiredo et al. ${ }^{5}$, no Paraná (Londrina), avaliaram 300 mulheres sem prolapso genital, com indicação de histerectomia por doença benigna, tendo realizado com sucesso histerectomia vaginal em 99\% (297 mulheres), com apenas duas conversões para laparotomia e uma para laparoscopia. O tempo cirúrgico médio foi de 51 minutos, com uma perda sanguínea média de $180 \mathrm{~mL}$ (variando entre 50 e $1050 \mathrm{~mL}$ ). Complicações intra-operatórias ocorreram em apenas quatro pacientes $(1,3 \%)$ e complicações pós-operatórias (infecção urinária) em 3,7\% (11 casos).

Não obstante todas essas vantagens da histerectomia vaginal, descritas em estudos observacionais, comparados ou não, deve-se salientar que, do ponto de vista da Medicina Baseada em Evidências, para nortear a decisão clínica e estabelecer novas práticas, essas não representam evidências de qualidade, uma vez que se categorizam em sua maioria como nivel IIb, grau de recomendação $B$. Inequivocamente, o melhor tipo de desenho de estudo para determinar a eficácia de uma intervenção é representado pelo ensaio clínico controlado, representando nível de evidência Ia ou Ib, grau de recomendação $\mathrm{A}^{23}$.

Nesse sentido, apesar de numerosas referências disponiveis na literatura sobre histerectomia vaginal, são muitos raros os ensaios clínicos randomizados comparando esta técnica às outras. Na verdade, pesquisando os bancos de dados LILACS/SciELO, Medline e EMBASE, encontramos apenas um ensaio clínico controlado comparando histerectomia vaginal simples (sem laparoscopia) com histerectomia abdominal $^{7}$ e dois ensaios clínicos comparando histerectomia vaginal com e sem laparoscopia ${ }^{11,24}$. Assim, até onde chega nosso conhecimento, este representa o segundo ensaio clínico controlado comparando histerectomia vaginal simples com a histerectomia abdominal, sendo certamente o primeiro realizado no Brasil. 
No estudo de Ottosen et al. ${ }^{7}$, realizado na Suécia, randomizaram-se 120 mulheres com indicação de histerectomia em três grupos: histerectomia vaginal simples, histerectomia vaginal assistida laparoscopicamente e histerectomia abdominal. O tempo cirúrgico foi significativamente maior para a histerectomia vaginal assistida laparoscopicamente (102 minutos) em relação à histerectomia vaginal simples (81 minutos) e à histerectomia abdominal (68 minutos). Apesar do tempo cirúrgico mais curto com a técnica abdominal, esta se associou a prolongada permanência hospitalar e maior tempo de convalescença. Estas duas variáveis não diferiram significativamente quando comparadas histerectomia vaginal simples e com laparoscopia. Os autores concluíram que a via vaginal deveria constituir a técnica de escolha para histerectomia.

Em outra direção, Summitt et al. ${ }^{11}$ compararam apenas os casos submetidos a histerectomia vaginal simples (27 casos) em relação à histerectomia vaginal assistida laparoscopicamente (29 casos). Encontraram um tempo cirúrgico significativamente menor para os casos de histerectomia vaginal isolada (65 minutos) em relação aos casos com laparoscopia (120 minutos), observando maior freqüência de dor pós-operatória e menores taxas de hematócrito nesse último grupo. Os custos da histerectomia assistida por laparoscopia foram significativamente maiores (em torno de US\$ 7900) do que os custos da cirurgia vaginal isolada (em torno de US\$4900). No outro ensaio clínico randomizado, Soriano et al. ${ }^{24}$ encontraram resultados semelhantes em termos de duração da cirurgia, porém não houve diferença na recuperação pós-operatória e duração de permanência hospitalar entre os dois grupos. Em ambos os estudos, os autores concluíram que não existem vantagens em se realizar a cirurgia vaginal combinada com laparoscopia.

Em suma, nosso estudo vem ao encontro dos resultados descritos por vários autores, tanto em estudos observacionais como em ensaios clínicos, apesar da raridade desses últimos. Julgamos que os resultados aqui encontrados são bastante encorajadores, animando-nos a prosseguir ampliando as indicações de histerectomia pela via vaginal em nosso serviço.

Um questionamento pertinente, portanto, é: por que ainda são realizadas tão poucas histerectomias por via vaginal em todo o mundo? De acordo com Davies et al. ${ }^{25}$, o treinamento e a experiência em cirurgia vaginal parecem ser os principais determinantes do tipo de cirurgia que se realiza nos diversos serviços. A maioria dos ginecologistas persiste relutante em realizar histerectomias vaginais em pacientes sem prolapso uterino, mesmo com evidências suficientes demonstrando a exeqüibilidade da cirurgia nessa condição ${ }^{7,25}$.

$\hat{E}$ indiscutivel que a histerectomia vaginal simples é menos invasiva do que a laparoscópica, uma vez que dispensa a incisão abdominal, pode ser realizada sem anestesia geral e com menor tempo cirúrgico, o que certamente minimiza os riscos operatórios. De acordo com Richardson et al. ${ }^{26}$, o uso da laparoscopia para a cirurgia vaginal representa na maior parte dos casos uma perda de tempo. Figueiredo et al. ${ }^{27}$ também discutem a necessidade de se utilizar o laparoscópio na histerectomia vaginal, uma vez que a cirurgia vaginal isolada é factivel na maior parte dos casos, sempre que o útero é móvel e não existe tumor anexial associado. Os autores sugerem que "em última análise, o principal papel do laparoscópio parece ser o de permitir que o ginecologista se dê conta de que a histerectomia vaginal simples pode ser realizada na maior parte dos casos".

Embora não disponhamos de dados brasileiros, devemos registrar que tem havido progressivo aumento do interesse pela histerectomia vaginal em nosso país, interesse este que pode ser avaliado pela disseminação de cursos sobre a técnica e pela abordagem cada vez mais freqüente do tema em congressos de Ginecologia e Obstetrícia.

A partir dos resultados encontrados, integrados às evidências disponiveis na literatura, julgamos válido recomendar que a via vaginal deve constituir a técnica de escolha, como primeira opção em pacientes com indicação de histerectomia, desde que presentes critérios como mobilidade uterina e volume uterino de no máximo 300 $\mathrm{cm}^{3}$. Evidentemente, com o aumento da experiência e habilidade dos cirurgiões, técnicas de morcelamento podem ser utilizadas para reduzir o volume uterino, ampliando portanto o leque de indicações da via vaginal, porém a comprovação da vantagem dessas técnicas requer outros ensaios clínicos controlados.

Julgamos ainda importante, no sentido de disseminar a prática da histerectomia vaginal em nossa região, uma maior ênfase em cirurgia vaginal nos programas de Residência Médica, bem como a reciclagem constante dos cirurgiões em atividade, em cursos com carga horária suficiente para o treinamento prático, sob supervisão de profissionais experientes. Por outro lado, devem ser conduzidos estudos nos diversos serviços, para determinar os resultados da adoção das novas práticas. Devemos salientar que a avaliação dos próprios resultados faz parte dos cinco passos recomendados para a prática da Medicina Baseada em Evidências, sendo fundamental que os diversos serviços gerem essas evidências ${ }^{28}$. 


\section{ABSTRACT}

Purpose: to compare intra- and postoperative results of vaginal hysterectomy with those of abdominal hysterectomy in women without genital prolapse or adnexal pathology.

Methods: a randomized, open clinical trial was conducted, involving 35 patients without genital prolapse scheduled for total hysterectomy due to benign disease, at IMIP, Recife, Brazil. These patients were randomly assigned to vaginal hysterectomy (19 patients) or abdominal hysterectomy (16 patients). Main outcome measures included estimated blood loss, rate of blood transfusion, duration of surgery, postoperative pain (intensity and analgesic requirement), time in hospital, postoperative complications, recovery time and patient satisfaction. Statistical analysis was performed using $\chi^{2}$, exact Fisher and Mann-Whitney tests at a $5 \%$ level of significance.

Results: estimated blood losses were significantly lower in vaginal hysterectomy (median of $520 \mathrm{~mL}$ ) than in abdominal hysterectomy (median $902 \mathrm{~mL}$ ). There was no blood transfusion among patients of the vaginal hysterectomy group, in contrast to $19 \%$ of the abdominal hysterectomy group. Duration of surgery was similar (median of $120 \mathrm{~min}$ in both groups). Postoperative pain, as measured by visual analog scale and analgesic requirement, was lower for vaginal hysterectomy than for abdominal hysterectomy. There was no statistically significant difference regarding frequency of postoperative complications. There was one case of infection in each group and one case of thrombosis in the vaginal hysterectomy group. Postoperative hospital stay was shorter in the vaginal group. Recovery time was significantly shorter in the vaginal group (median of 35 days) versus the abdominal group (median 40 days). Overall patient satisfaction with the operation was similar in the two groups. Conclusions: patients without genital prolapse submitted to vaginal hysterectomy for treatment of benign diseases had some advantages in relation to those submitted to abdominal hysterectomy: lower intraoperative blood loss, lower postoperative pain and faster recovery time. Vaginal hysterectomy may replace abdominal hysterectomy in most patients who require hysterectomy.

KEYWORDS: Abdominal hysterectomy. Vaginal hysterectomy. Gynecologic surgery. Postoperative complications. Clinical trial.

\section{Referências}

1. Wilcox LS, Koonin LM, Pokras R, Strauss LT, Xia Z, Peterson HB. Hysterectomy in the United States, 1988-1990. Obstet Gynecol 1994; 83:549-55.

2. Ministério da Saúde. DATASUS. Informações de Saúde. Sistema de Informações Hospitalares do SUS. Brasília; 2002.

3. Camargo SF, Ribeiro ACS. Indicações atuais da cirurgia vaginal: experiência brasileira. Femina 2001; 29:393-4.
4. Macedo ALS, Simões PM, Macedo FLS. Histerectomia vaginal em pacientes não portadoras de prolapso do útero: estudo de 370 casos. Rev Bras Ginecol Obstet 1995; 17:1011-9.

5. Figueiredo O, Figueiredo EG, Figueiredo PG, Pelosi MA. Vaginal removal of the benign nonprolapsed uterus: experience with 300 consecutive operations. Obstet Gynecol 1999; 94:348-51.

6. Reich H, De Caprio J, McGlynn F. Laparoscopic hysterectomy. J Gynecol Surg 1989; 5: 313-216.

7. Ottosen C, Lingman G, Otossen L. Three methods for hysterectomy: a randomised, prospective study of short-term outcome. BJOG 2000; 107:1380-5.

8. Dicker RC, Greenspan JR, Strauss LT, et al. Complications of abdominal and vaginal hysterectomy among women of reproductive age in the United States. The Collaborative Review of Sterilization. Am J Obstet Gynecol 1982; 144:841-8.

9. Nezhat C, Bess O, Admon D, Nezhat CH, Nezhat F. Hospital cost comparison between abdominal, vaginal, and laparoscopy-assisted vaginal hysterectomies. Obstet Gynecol 1994; 83:713-6.

10.Dorsey JH, Holtz PM, Griffiths RI, McGrath MM, Steinberg EP. Costs and charges associated with three alternative techniques of hysterectomy. N Engl J Med 1996; 335:476-82

11.Summitt RL Jr, Stovall TG, Lipscomb GH, Ling FW. Randomized comparison of laparoscopy-assisted vaginal hysterectomy with standard vaginal hysterectomy in an outpatient setting. Obstet Gynecol 1992; 80:895-901.

12.Raju KS, Auld BJ. A randomised prospective study of laparoscopic vaginal hysterectomy versus abdominal hysterectomy each with bilateral salpingo-oophorectomy. Br J Obstet Gynaecol 1994; 101:1068-71.

13.Falcone T, Paraiso MF, Mascha E. Prospective randomized clinical trial of laparoscopically assisted vaginal hysterectomy versus total abdominal hysterectomy. Am J Obstet Gynecol 1999; 180:955-62.

14.Marana R, Busacca M, Zupi E, Garcea N, Paparella P, Catalano GF. Laparoscopically assisted vaginal hysterectomy versus total abdominal: a prospective, randomized, multicenter study. Am J Obstet Gynecol 1999; 180:270-5.

15.Amorim MMR, Costa AAR, Santos LC, Barradas P, da Hora AMT. Histerectomia vaginal sem prolapso: experiência inicial no IMIP. Acta Cir Bras 2002; 17 (Supl 1):58.

16. Heaney NS. Vaginal hysterectomy: its indications and technique. Am J Surg 1940; 48:284-8.

17. Richardson EH. A simplified technique for abdominal panhysterectomy. Surg Gynecol Obstet 1929; 48:248-56. 
18. Mangram AJ, Horan TC, Pearson ML, Silver LC, Jarvis WR. Guideline for Prevention of Surgical Site Infection, 1999. Centers for Disease Control and Prevention (CDC) Hospital Infection Control Practices Advisory Committee. Am J Infect Control 1999; 27:97-132.

19.Cosson M, Lambaudie E, Boukerrou M, Querleu D, Crépin G. Vaginal, laparoscopic, or abdominal hysterectomies for benign disorders: immediate and early postoperative complications. Eur J Obstet Gynecol Reprod Biol 2001; 98:231-6.

20.Kovac SR. Hysterectomy outcomes in patients with similar indications. Obstet Gynecol 2000; 95:787-93.

21.Varma R, Tahseen S, Lokugamage AU, Kunde D. Vaginal route as the norm when planning hysterectomy for benign conditions: charge in practice. Obstet Gynecol 2001; 97:613-6.

22.Cosson M, Rajabally R, Querleu D, Crepin G. Long term complications of vaginal hysterectomy: a case control study. Eur J Obstet Gynecol Reprod Biol 2001; 94:239-44.
23. Meakins JL. Innovation in surgery: the rules of evidences. Am J Surg 2002; 183:399-405.

24.Soriano D, Goldstein A, Lecuru F, Darai E. Recovery from vaginal hysterectomy compared with laparoscopy-assisted vaginal hysterectomy: a prospective, randomized, multicenter study. Acta Obstet Gynecol Scand 2001; 80:337-41.

25.Davies A, Vizza E, Bournas N, O`Connor H, Magos A. How to increase the proportion of hysterectomies performed vaginally. Am J Obstet Gynecol 1998; 179:1008-12.

26. Richardson RE, Bournas N, Magos AL. Is laparoscopic hysterectomy waste of time? Lancet 1995 ; 345:36-41.

27.Figueiredo Netto O, Figueiredo O, Figueiredo EG, Figueiredo $P G$ Histerectomia vaginal: o laparoscópico é necessário? Rev Bras Ginecol Obstet 1998; 20:537-40.

28.Sackett DL, Straus SE, Richardson WS, Rosenberg W, Haynes RB. Evidence-based medicine. How to practice and teach EBM. $2^{\text {nd }}$ ed. Edinburgh: Churchill Livingstone; 2000. p.261.

\section{OURSOSPREIARATÓRIOS PARAOTEGO}

Tendo em vista que diversos cursos estão fazendo propaganda como sendo preparatórios para o TEGO, a FEBRASGO alerta aos candidatos que não mantém com eles qualquer vinculação, não existindo nenhum curso que tenha sido credenciado ou de qualquer forma autorizado pela FEBRASGO a ministrar curso daquela natureza.

A FEBRASGO alerta, também, que a sua Diretoria vedou a participação de qualquer membro da Comissão Nacional do TEGO de colaborar com tais cursos, inclusive com a elaboração de apostilas, sob pena de caracterizar comportamento contrário à ética, a ser apurado em processo disciplinar.

A FEBRASGO permanece à disposição dos candidatos para prestar esclarecimentos sobre o TEGO, bem como para receber denúncia relativa aos fatos acima, de forma que não haja dúvida sobre o seu comprometimento com a lisura do concurso, em todos os seus aspectos. 